\title{
RIGIDITY OF MINIMIZERS IN NONLOCAL PHASE TRANSITIONS
}

\author{
O. SAVIN
}

\begin{abstract}
We obtain the classification of certain global bounded solutions for semilinear nonlocal equations of the type

$$
\triangle^{s} u=W^{\prime}(u) \quad \text { in } \quad \mathbb{R}^{n}, \quad \text { with } \quad s \in(1 / 2,1),
$$

where $W$ is a double well potential.
\end{abstract}

\section{INTRODUCTION}

In this article we extend to the case of the fractional Laplacian $\triangle^{s}$ with $s \in$ $(1 / 2,1)$ the results from [S1, [S2] concerning a conjecture of De Giorgi about the classification of certain global bounded solutions for semilinear equations of the type

$$
\triangle u=W^{\prime}(u),
$$

where $W$ is a double well potential.

We consider the Ginzburg-Landau energy functional with nonlocal interactions

$$
J(u, \Omega)=\frac{1}{4} \int_{\mathbb{R}^{n} \times \mathbb{R}^{n} \backslash(\mathcal{C} \Omega \times \mathcal{C} \Omega)} \frac{(u(x)-u(y))^{2}}{|x-y|^{n+2 s}} d x d y+\int_{\Omega} W(u) d x,
$$

with $|u| \leq 1$. Here $W$ is a double-well potential with minima at 1 and -1 satisfying

$$
\begin{gathered}
W \in C^{2}([-1,1]), \quad W(-1)=W(1)=0, \quad W>0 \text { on }(-1,1), \\
W^{\prime}(-1)=W^{\prime}(1)=0, \quad W^{\prime \prime}(-1)>0, \quad W^{\prime \prime}(1)>0 .
\end{gathered}
$$

The classical double-well potential $W$ to have in mind is

$$
W(s)=\frac{1}{4}\left(1-s^{2}\right)^{2} .
$$

Physically $u \equiv-1$ and $u \equiv 1$ represent the stable "phases". A critical function for the energy $J$ corresponds to a phase transition with nonlocal interaction between these states, and it satisfies the Euler-Lagrange equation

$$
\triangle^{s} u=W^{\prime}(u)
$$

where $\triangle^{s} u$ is defined as

$$
\triangle^{s} u(x)=P V \int_{\mathbb{R}^{n}} \frac{u(y)-u(x)}{|y-x|^{n+2 s}} d y .
$$

Our main result provides the classification of minimizers with asymptotically flat level sets.

Theorem 1.1. Let $u$ be a global minimizer of $J$ in $\mathbb{R}^{n}$ with $s \in\left(\frac{1}{2}, 1\right)$. If the 0 level set $\{u=0\}$ is asymptotically flat at $\infty$, then $u$ is one-dimensional. 
A more quantitative version of Theorem 1.1 is given in Theorem 6.1.

In a subsequent work we will treat also the case $s=\frac{1}{2}$ which requires some modifications of the methods presented in this paper. We remark that Theorem 1.1 when $s \in\left(0, \frac{1}{2}\right)$ was obtained recently by Dipierro, Valdinoci and Serra [DVS.

It is known that blowdowns of the level set $\{u=0\}$ have different behavior depending on the value of $s$. If $s \geq 1 / 2$, there are sequences $\varepsilon_{k}\{u=0\}$ with $\varepsilon_{k} \rightarrow 0$ that converge uniformly on compact sets to a minimal surface and, if $s<1 / 2$ they converge to a $s$-nonlocal minimal surface. This follows from a $\Gamma$-convergence result together with a uniform density estimate of level sets of minimizers which were obtained by the author and Valdinoci in SV1, SV2, see for example Corollary 1.7 in SV1.

From the classification of global minimal surfaces in low dimensions we find that the level sets of minimizers of $J$ are always asymptotically flat at $\infty$ in dimension $n \leq 7$ if $s \geq 1 / 2$, and we obtain the following corollary of Theorem 1.1.

Theorem 1.2. A global minimizer of $J$ is one-dimensional in dimension $n \leq 7$ if $s \in\left(\frac{1}{2}, 1\right)$.

Another consequence of Theorem 1.1 is the following version of De Giorgi's conjecture to the fractional Laplace case.

Theorem 1.3. Let $u \in C^{2}\left(\mathbb{R}^{n}\right)$ be a solution of

$$
\triangle^{s} u=W^{\prime}(u),
$$

with $s \in(1 / 2,1)$, such that

$$
|u| \leq 1, \quad \partial_{n} u>0, \quad \lim _{x_{n} \rightarrow \pm \infty} u\left(x^{\prime}, x_{n}\right)= \pm 1 .
$$

Then $u$ is one-dimensional if $n \leq 8$.

Theorem 1.2 and Theorem 1.3 without the limit assumption in (1.2) have been proved in 2 and 3 dimensions using stability inequality methods. In dimension $n=3$ and for $s \geq 1 / 2$ they have been established by Cabre and Cinti CC2 , and in dimension $n=2$ for all $s \in(0,1)$ by Sire and Valdinoci [SiV], see also [CC1], CS2, $\mathrm{CSO}$.

It is not difficult to show that the \pm 1 limit assumption implies that $u$ is a global minimizer in $\mathbb{R}^{n}$, see for example Theorem 1 in PSV]. Since $\{u=0\}$ is a graph, it is asymptotically flat in dimension $n \leq 8$ and Theorem 1.1 applies.

Similarly we see that if the 0 level set is a graph in the $x_{n}$ direction which has a one sided linear bound at $\infty$ then the conclusion is true in any dimension.

Theorem 1.4. If $u$ satisfies (1.1), (1.2), and

$$
\{u=0\} \subset\left\{x_{n}<C\left(1+\left|x^{\prime}\right|\right)\right\},
$$

and $s \in\left(\frac{1}{2}, 1\right)$ then $u$ is one-dimensional.

Our proof of Theorem 1.1 follows closely the one for the classical Laplacian given in [S2]. The main steps consist in 1) finding some appropriate families of radial subsolutions, 2) applying a version of weak Harnack inequality and 3) a $\Gamma$-convergence result. Some new technicalities are present in our setting due to the nonlocal nature of the equation. For example in the improvement of flatness property Theorem 6.1, we need to impose a geometric restriction to the level set $\{u=0\}$ possibly outside the flat cylinder $\mathcal{C}(l, \theta)$. 
We prove Theorem 1.1 by making use of the extension property of the fractional Laplacian of Caffarelli-Silvestre [CS]. Precisely we consider the extension $U(x, y)$ of $u(x)$ in $\mathbb{R}_{+}^{n+1}$ such that

$$
\operatorname{div}\left(y^{a} \nabla U\right)=0 \quad \text { in } \mathbb{R}_{+}^{n+1}, \quad U(x, 0)=u(x), \quad a:=1-2 s \in(-1,1),
$$

and then

$$
\triangle^{s} u(x)=c_{n, s} \lim _{y \rightarrow 0^{+}} y^{a} U_{y}(x, y),
$$

with $c_{n, s}$ a constant that depends only on $n$ and $s$. Then global minimizers of $J(u)$ in $\mathbb{R}^{n}$ with $|u| \leq 1$ correspond to global minimizers of the "extension energy" $\mathcal{J}(U)$ with $|U| \leq 1$ where

$$
\mathcal{J}(U):=\frac{c_{n, s}}{2} \int|\nabla U|^{2} y^{a} d x d y+\int W(u) d x .
$$

After dividing by a constant and relabeling $W$ we may fix $c_{n, s}$ to be 1 . We obtain an improvment of flatness property for the level sets of minimizers of $\mathcal{J}$ which are defined in large balls $\mathcal{B}_{R}^{+}$, see Theorem 6.1. We remark that the principal use of the extension is to make the various subsolution computations easier to handle and it is not essential to the method of proof.

The paper is organized as follows. In Sections 2 and 3 we introduce some notation and then construct a family of axial subsolutions. In Section 4 we provide certain "viscosity solution" properties of the level set $\{u=0\}$. In Section 5 we obtain a Harnack inequality of the 0 level set and in Section 6 we prove Theorem6.1.

\section{NOTATION AND PRELIMINARIES}

We introduce the following notation:

We denote points in $\mathbb{R}^{n}$ as $x=\left(x^{\prime}, x_{n}\right)$ with $x^{\prime} \in \mathbb{R}^{n-1}$. The ball of center $z$ and radius $r$ is denoted by $B_{r}(z)$,

$$
B_{r}(z):=\left\{x \in \mathbb{R}^{n}|| x-z \mid<r\right\}, \quad B_{r}:=B_{r}(0) .
$$

The cylinder with base $l$ and height $\theta$ is denoted by $\mathcal{C}(l, \theta) \subset \mathbb{R}^{n}$

$$
\mathcal{C}(l, \theta):=\left\{x|\quad| x^{\prime}|\leq l, \quad| x_{n} \mid \leq \theta\right\} .
$$

Points in the extension variables $\mathbb{R}_{+}^{n+1}$ are denoted by $(x, y)$ with $y>0$, and the ball of radius $r$ as $\mathcal{B}_{r}^{+}$

$$
\mathcal{B}_{r}^{+}:=\left\{(x, y) \in \mathbb{R}_{+}^{n+1}|\quad|(x, y) \mid<r\right\} \quad \subset \mathbb{R}^{n+1} .
$$

Given a function $U(x, y)$ we define $u$ its trace on $\{y=0\}$

$$
u(x)=U(x, 0) .
$$

Also let

and

$$
a:=1-2 s \in(-1,0),
$$

$$
\begin{gathered}
\triangle_{a} U:=\triangle U+a \frac{U_{y}}{y}=y^{-a} \operatorname{div}\left(y^{a} \nabla U\right), \\
\partial_{y}^{1-a} U(x):=\lim _{y \rightarrow 0^{+}} y^{a} U_{y}(x, y)=\frac{1}{1-a} \lim _{y \rightarrow 0^{+}} y^{a-1}(U(x, y)-U(x, 0)) .
\end{gathered}
$$

We define the energy $\mathcal{J}$ as

$$
\mathcal{J}\left(U, \mathcal{B}_{R}^{+}\right):=\frac{1}{2} \int_{\mathcal{B}_{R}^{+}}|\nabla U|^{2} y^{a} d x d y+\int_{B_{r}} W(u) d x,
$$


and a critical function $U$ for $\mathcal{J}$ satisfies the Euler-Lagrange equation

$$
\triangle_{a} U=0, \quad \partial_{y}^{1-a} U=W^{\prime}(u) .
$$

In [PSV] Theorem 2, see also [CS1], it was proved the existence and uniqueness up to translations of a global minimizer of $\mathcal{J}$ in $2 \mathrm{D}$ which is increasing in the first variable and which has limits \pm 1 at infinity. Precisely there exists a unique $G: \mathbb{R}_{+}^{2} \rightarrow(-1,1)$ that solves the equation (2.1) such that $G(t, y)$ is increasing in the $t$ variable and its trace $g(t):=G(t, 0)$ satisfies

$$
g(0)=0, \quad \lim _{t \rightarrow \pm \infty} g(t)= \pm 1 .
$$

Moreover, $g$ and $g^{\prime}$ have the following asymptotic behavior

$$
1-|g| \sim \min \left\{1,|t|^{-2 s}\right\}, \quad g^{\prime} \sim \min \left\{1,|t|^{-1-2 s}\right\},
$$

and since $a \in(-1,0)$ we have $\mathcal{J}\left(G, \mathbb{R}_{+}^{2}\right)<\infty$.

Since $\triangle_{a} G_{t}=0$ and $G_{t} \geq 0$, we easily conclude that

$$
|\nabla G| \leq C \min \left\{1, r^{-1}\right\}, \quad G_{t} \geq c r^{-1-2 s} .
$$

where $r$ denotes the distance to the origin in the $(t, y)$-plane.

In Theorem 6.1 we show that the only global minimizer of $\mathcal{J}$ that has asymptotically flat level sets on $y=0$ is $G\left(x_{n}, y\right)$ up to translations and rotations.

For simplicity of notation we assume that $W$ is uniformly convex outside the interval $[g(-1), g(1)]$.

Constants that depend on $n, s, W, G$ are called universal constants, and we denote them by $C, c$. In the course of the proofs the values of $C, c$ may change from line to line when there is no possibility of confusion. If the constants depend on other parameters, say $\theta, \rho$, then we denote them by $C(\theta, \rho)$ etc.

\section{2D BARRIERS}

In this section we construct two families of comparison functions $G_{R}$ and $\Psi_{R}$ which are perturbations of the solution $G$.

Lemma 3.1 (Radial subsolutions). For all large $R$, there exist continuous functions $G_{R}: \mathbb{R}^{2} \rightarrow(-1,1]$, and $\delta>0$ small, $C$ large universal constants such that:

1) $G_{R}=1$ outside $\mathcal{B}_{R^{1-\delta}}^{+} \cup\left((-\infty, 0] \times\left[0, R^{1-\delta}\right]\right)$,

2) $G_{R}(t, y)$ is nondecreasing in $t$, and $\partial_{t} G_{R}=0$ outside $\mathcal{B}_{R^{1-\delta}}^{+}$,

3)

$$
\left|G_{R}-G\right| \leq \frac{C}{R} \quad \text { in } \mathcal{B}_{4}^{+}
$$

4)

$$
\triangle_{a} G_{R}+\frac{2(n-1)}{R}\left|\nabla G_{R}\right| \leq 0
$$

and on $y=0$ :

$$
\partial_{y}^{1-a} G_{R}<W^{\prime}\left(G_{R}\right) \quad \text { if } t \notin[-1,1] .
$$


The inequalities in 4) are understood in the viscosity sense.

Notice that by (2.2), property 3) implies that

$$
G_{R}(t, y) \leq G\left(t+\frac{C^{\prime}}{R}, y\right) \quad \text { in } \quad \mathcal{B}_{4}^{+} .
$$

We remark that property 3 ) and the inequality above hold in any ball $\mathcal{B}_{K}^{+}$, for a fixed large constant $K$, provided that we replace $C / R, C^{\prime} / R$ by $C(K) / R, C^{\prime}(K) / R$.

Proof. We begin with the following claim whose proof we provide at the end.

Claim: For each $\alpha \in(1,1-a)$ there exists $H$ a homogenous of degree $\alpha$ function such that

$$
H \geq r^{\alpha}, \quad \triangle_{a} H \leq-r^{\alpha-2}, \quad|\nabla H| \leq C r^{\alpha-1}, \quad \partial_{y}^{1-a} H \leq C|t|^{\alpha-(1-a)} .
$$

Here $r$ denotes the distance to the origin and $C=C(\alpha)$ depends on the universal constants and $\alpha$.

Fix such an $\alpha$ and define

$$
H_{R}:=\min \left\{G+\frac{C_{0}}{R}\left(H+C_{1}\right), \quad 1\right\},
$$

with $C_{0}, C_{1}$ large constants to be specified later.

We define $G_{R}$ as the infimum over all left translations of $H_{R}$ i.e.

$$
G_{R}(t, y)=\inf _{l \geq 0} H_{R}(t+l, y) .
$$

Since $|G|<1$ we have $H_{R}>-1$, and $H_{R}=1$ outside $\mathcal{B}_{R^{1-\delta}}^{+}$provided that $\delta$ is chosen sufficiently small such that $(1-\delta) \alpha>1$. Properties 1$)$ and 2) are clearly satisfied.

Notice that $H$ is increasing in a band $[C, \infty) \times[0,4]$ and we obtain that $H_{R}$ is increasing in $[-4, \infty) \times[0,4]$. This gives $G_{R}=H_{R}$ in $\mathcal{B}_{4}^{+}$and property 3) is satisfied.

The properties of $H$ and (2.2) imply that in the set $\left\{H_{R}<1\right\}$ we have

$$
\left|\nabla H_{R}\right| \leq C \min \left\{1, r^{-1}\right\}+C C_{0} R^{-1} r^{\alpha-1},
$$

and

$$
\triangle_{a} H_{R} \leq-C_{0} R^{-1} r^{\alpha-2} .
$$

Then the first inequality in 4) holds for $H_{R}$ provided that $C_{0}$ is chosen sufficiently large, and therefore holds also for $G_{R}$ as the infimum over translations of $H_{R}$.

On $y=0$ in the set $\left\{H_{R}<1\right\}$ we have

$$
\partial_{y}^{1-a} H_{R}=\partial_{y}^{1-a} G+C_{0} R^{-1} \partial_{y}^{1-a} H \leq W^{\prime}(G)+C R^{-1}|t|^{\alpha-(1-a)} .
$$

From the behavior of $g$ and $g^{\prime}$ for large $t$, we see that the minimum of $H_{R}(t, 0)$ occurs at some $t=q_{R} \sim-R^{1 /(2 s+\alpha)} \ll-1$ and

$$
\left\|\left(H_{R}-G\right)(t, 0)\right\|_{L^{\infty}\left(\left[q_{R}, \infty\right)\right.} \rightarrow 0 \quad \text { as } \quad R \rightarrow \infty .
$$

Since $W^{\prime \prime} \geq c$ outside $[g(-1), g(1)]$ we find that when $t \in\left[q_{R}, \infty\right) \backslash[-1,1]$ and $\left\{H_{R}<1\right\}$ we have

$$
W^{\prime}\left(H_{R}\right)-W^{\prime}(G) \geq \frac{c}{2}\left(H_{R}-G\right) \geq c^{\prime} R^{-1}\left(|t|^{\alpha}+C_{1}\right),
$$

thus, if $C_{1}$ is sufficiently large,

$$
\partial_{y}^{1-a} H_{R}<W^{\prime}\left(H_{R}\right) \quad \text { in } \quad\left[q_{R}, \infty\right) \backslash[-1,1]
$$


Now the second inequality of 4$)$ is satisfied by $G_{R}$ as the infimum of left translations of $H_{R}$.

Proof of Claim: We find $H$ as a perturbation of the function $C y^{\alpha}$ near $y=0$. Notice that $y^{1-a}$ is $\triangle_{a}$-harmonic, thus $y^{\alpha}$ is $\triangle_{a}$-superharmonic for $\alpha<1-a$. However $C y^{\alpha}$ does not satisfy the first and the third property given in the claim.

We write $H$ in polar coordinates as, $H=r^{\alpha} h(\theta)$ with $h$ an even function with respect to $\pi / 2$ and then

$$
\begin{gathered}
r^{2-\alpha} \triangle_{a} H=h^{\prime \prime}+\alpha(\alpha+a) h+a \cot \theta h^{\prime}, \\
\partial_{y}^{1-a} H=r^{\alpha-(1-a)} \partial_{\theta}^{1-a} h .
\end{gathered}
$$

For all small $\sigma$, the function

$$
h_{\sigma}=\sigma+\theta^{1-a}-\theta^{2},
$$

gives a negative right hand side in (3.2) when $\theta$ belongs to a small fixed interval $[0, c]$. We choose first $M$ large and then $\sigma$ small such that the graphs of $M h_{\sigma}$ and $(\sin \theta)^{\alpha}$ become tangent by above at some point in the interval $[0, c]$. Now we "glue" parts of the two graphs in a single graph of a $C^{1,1}$ function $\tilde{h}$. Now it is easy to check that all properties hold by taking $h$ a large multiple of $\tilde{h}$.

From the construction of $H_{R}, G_{R}$ we see that both of them decrease with $R$ as we increase $R$.

Next we construct a similar family $\Psi_{R}$ which can be compared with $G_{\bar{R}}$ even when $R$ and $\bar{R}$ have different orders of magnitude.

Lemma 3.2. There exist functions $G_{R}$ and $\Psi_{R}$ that satisfy the properties 1)-4) of Lemma 3.1 for some $\delta, C$ universal such that

$$
G_{R}\left(t+R^{-\sigma}, y\right) \geq \Psi_{R^{1-\sigma}}(t, y),
$$

with $\sigma \in(0, \delta / 3)$ small universal.

Proof. Denote by $G_{R, \alpha}$ the function constructed in Lemma 3.1 .

We choose $G_{R}:=G_{R, \alpha}, \Psi_{R}:=G_{R, \beta}$ for some fixed $\alpha, \beta$ such that $1<\beta<\alpha<$ $1-a$. We take $\delta=\min \{\delta(\alpha), \delta(\beta)\}$, and $C=\max \{C(\alpha), C(\beta)\}$ and then Lemma 3.1 holds for both $G_{R}$ and $\Psi_{R}$ with the same constants $\delta$ and $C$.

We show that

$$
H_{R, \alpha}\left(t+R^{-\sigma}, y\right) \geq H_{R^{1-\sigma}, \beta}(t, y),
$$

with $H_{R, \alpha}$ defined as in (3.1), and the lemma follows by taking the infimum over the left translations.

In the inequality above it suffices to restrict to the set where $\left\{H_{R, \alpha}<1\right.$. $\}$ We have

$$
H_{R} \geq G+R^{-1}\left(c_{1} r^{\alpha}+c_{2}\right),
$$

for some constants $c_{1}, c_{2}$ depending on $\alpha$. After a translation of $R^{-\sigma}$ we obtain $($ see $(2.2)$ )

$$
H_{R}\left(t+R^{-\sigma}, y\right) \geq G(t, y)+c R^{-\sigma} \min \left\{1, r^{-1-2 s}\right\}+\frac{1}{2} R^{-1}\left(c_{1} r^{\alpha}+c_{2}\right) .
$$

When $r \geq 1$ we use the inequality $a+b \geq a^{\mu} b^{1-\mu}$ for $\mu>0$ small, and we find

$$
H_{R}\left(t+R^{-\sigma}, y\right) \geq G(t, y)+c(\alpha) R^{-\eta}\left(r^{\gamma}+1\right),
$$


with

$$
\gamma=\alpha(1-\mu)-\mu(1+2 s), \quad \eta=1-\mu+\sigma \mu
$$

(and $\eta>\sigma$.) We choose $\mu$ small and then $\sigma$ such that $\gamma>\beta$ and $\eta<1-\sigma$. Then the right hand side of (3.4) is grater than

$$
G+R^{\sigma-1}\left(C_{1}(\beta) r^{\beta}+C_{2}(\beta)\right) \geq H_{R^{1-\sigma}, \beta},
$$

for all large $R$, and the lemma is proved.

Remark 3.3. Using the monotonicity of $\Psi_{r}$ with respect to $r$, we have

$$
G_{R}\left(s+R^{-\sigma}, y\right) \geq \Psi_{r}(s, y), \quad \forall r \geq R^{1-\sigma} .
$$

\section{Estimates FOR $\{u=0\}$}

In this section we derive properties of the level sets of solutions to

$$
\triangle_{a} U=0, \quad \partial_{y}^{1-a} U=W^{\prime}(U),
$$

which are defined in large domains.

In the next lemma we find axial approximations to the $2 \mathrm{D}$ solution $G$.

Lemma 4.1 (Axial approximations). Let $G_{R}: \mathbb{R}_{+}^{2} \rightarrow(-1,1]$ be the function constructed in Lemma 3.2. Then its axial rotation in $\mathbb{R}^{n+1}$

$$
\Phi_{R}(x, y):=G_{R}(|x|-R, y)
$$

satisfies

1) $\Phi_{R}=1$ outside $\mathcal{B}_{R+R^{1-\delta}}^{+}$,

2)

and

$$
\triangle_{a} \Phi_{R} \leq 0 \quad \text { in } \quad \mathbb{R}_{+}^{n+1}
$$

$$
\partial_{y}^{1-a} \Phi_{R}<W^{\prime}\left(\Phi_{R}\right) \quad \text { when } \quad|x|-R \notin[-1,1] .
$$

Let $\phi_{R}(x)=\Phi_{R}(x, 0)$ denote the trace of $\Phi_{R}$ on $\{y=0\}$. Notice that $\phi_{R}$ is radially increasing, and $\left\{\phi_{R}=0\right\}$ is a sphere which is in a $C / R$-neighborhood of the sphere of radius $R$.

Proof. We have

$$
\begin{gathered}
\triangle_{a} \Phi_{R}(x, y)=\triangle_{a} G_{R}(s, y)+\frac{n-1}{R+s} \partial_{s} G_{R}(s, y), \quad s=|x|-R, \\
\partial_{y}^{1-a} \Phi_{R}(x, 0)=\partial_{y}^{1-a} G_{R}(s, 0) .
\end{gathered}
$$

The conclusion follows from Lemma 3.2 since $\partial_{s} G_{R}=0$ when $|s| \geq R^{1-\delta}$ and $R+s>R / 2$ when $|s|<R^{1-\delta}$.

Definition 4.2. We denote by $\Phi_{R, z}$ the translation of $\Phi_{R}$ by $z$ i.e.

$$
\Phi_{R, z}(x, y):=\Phi_{R}(x-z, y)=G_{R}(|x-z|-R, y) .
$$

Similarly we define $\Psi_{R, z}$ the axial rotation of the other $2 \mathrm{D}$ solution $\Psi_{R}$ given in Lemma 3.2 .

$$
\Psi_{R, z}(x, y):=\Psi_{R}(|x-z|-R, y) .
$$

Clearly $\Psi_{R, 0}$ satisfies properties 1), 2) of Lemma 4.1. 
Sliding the graph of $\Phi_{R}$ :

Assume that $u$ is less than $\phi_{R, x_{0}}$ in $B_{2 R}\left(x_{0}\right)$. By the maximum principle we obtain that $U<\Phi_{R, z}$ with $z=x_{0}$ in $\mathcal{B}_{2 R}\left(x_{0}, 0\right)$ (and therefore globally.) We translate the function $\Phi_{R}$ above by moving continuously the center $z$, and let's assume that it touches $U$ by above, say for simplicity when $z=0$, i.e. the strict inequality becomes equality for some contact point $\left(x^{*}, y^{*}\right)$. From Lemma 4.1 we know that $\Phi_{R}$ is a strict supersolution away from $\{y=0\}$, and moreover the contact point must satisfy $y^{*}=0,\left|x^{*}\right|-R \in[-1,1]$, that is it belongs to the annular region $B_{R+1} \backslash B_{R-1}$ in the $n$-dimensional subspace $\{y=0\}$.

Lemma 4.3 (Estimates near a contact point). Assume that the graph of $\Phi_{R}$ touches by above the graph of $U$ at a point $\left(x^{*}, 0, u\left(x^{*}\right)\right)$ with $x^{*} \in B_{R+1} \backslash B_{R-1}$. Let $\pi\left(x^{*}\right)$ be the projection of $x^{*}$ onto the sphere $\partial B_{R}$. Then in $\mathcal{B}_{1}\left(\pi\left(x^{*}\right), 0\right)$

1) $\{u=0\}$ is a smooth hypersurface in $\mathbb{R}^{n}$ with curvatures bounded by $\frac{C}{R}$ which stays in a $\frac{C}{R}$ neighborhood of $\partial B_{R}$.

2)

$$
|U-G(x \cdot \nu-R, y)| \leq \frac{C}{R}, \quad \nu:=\pi\left(x^{*}\right) / R .
$$

Proof. Assume for simplicity that $x^{*}$ is on the positive $x_{n}$ axis and therefore $\pi\left(x^{*}\right)=$ $R e_{n},\left|x^{*}-R e_{n}\right| \leq 1$. By Lemma 4.1 we have

$$
U \leq \Phi_{R} \leq G\left(|x|-R+\frac{C}{R}, y\right) \leq G\left(x_{n}-R+\frac{C^{\prime}}{R}, y\right)=: V \quad \text { in } \quad \mathcal{B}_{3}\left(R e_{n}\right) .
$$

Both $U$ and $V$ solve the same equation (4.1), and

$$
(V-U)\left(x^{*}, 0\right) \leq \frac{C^{\prime \prime}}{R}
$$

Since $V-U \geq 0$ satisfies

$$
\begin{aligned}
\triangle_{a}(V-U) & =0, \quad \partial_{y}^{1-a}(V-U)=b(x)(V-U), \\
b(x) & :=\int_{0}^{1} W^{\prime \prime}(t u(x)+(1-t) v(x)) d t,
\end{aligned}
$$

we obtain

$$
|V-U| \leq \frac{C}{R} \quad \text { in } \quad \mathcal{B}_{5 / 2}\left(R e_{n}\right),
$$

from the Harnack inequality with Neumann condition for $\triangle_{a}$. Moreover since $b$ has bounded Lipschitz norm and $s>1 / 2$ we obtain that $U-V \in C_{x}^{2, \alpha}$ for some $\alpha>0$, and

$$
\|U-V\|_{C_{x}^{2, \alpha}\left(\mathcal{B}_{2}\left(R e_{n}\right)\right)} \leq \frac{C}{R}
$$

by local Schauder estimates. This easily implies the lemma.

Remark 4.4. If instead of $\mathcal{B}_{1}\left(\left(\pi\left(x^{*}\right), 0\right)\right)$ we write the conclusion in $\mathcal{B}_{K}\left(\left(\pi\left(x^{*}\right), 0\right)\right)$ for some large, fixed constant $K$, then we need to replace $\frac{C}{R}$ by $\frac{C(K)}{R}$. Here $C(K)$ represents a constant which depends also on $K$.

Next we obtain estimates near a point on $\{u=0\}$ which admits a one-sided tangent ball of large radius $R$. 
Lemma 4.5. Assume that $U$ is defined in $\mathcal{B}_{2 R}^{+}$, satisfies (4.1), and that

a) $B_{R}\left(-R e_{n}\right) \subset\{u<0\}$ is tangent to $\{u=0\}$ at 0 ,

b) there is $x_{0} \in B_{R / 2}\left(-R e_{n}\right)$ such that $u\left(x_{0}\right) \leq-1+c$ for some $c>0$ small. Then

1) $\{u=0\}$ is smooth in $B_{1}$ and has curvatures bounded by $\frac{C}{R}$.

2) $\left|U-G\left(x_{n}, y\right)\right| \leq \frac{C}{R}$ in $\mathcal{B}_{1}$.

Proof. Assume first that $u<\phi_{R / 8, z}$ for $z=-R e_{n}$.

We translate the graph of $\Phi_{R / 8, z}$ by moving $z$ continuously upward on the $x_{n}$ axis. We stop when the translating graph becomes tangent by above to the graph of $U$ for the first time. Denote by $\left(x^{*}, 0, u\left(x^{*}\right)\right)$ the contact point and by $z^{*}$ the final center $z$ and by $\pi\left(x^{*}\right)$ the projection of $x^{*}$ onto $\partial B_{R / 8}\left(z^{*}\right)$.

By Lemma 4.3, $\{u=0\}$ must be in a $\frac{C_{1}}{R}$ neighborhood of $\partial B_{R / 8}\left(z^{*}\right) \cap B_{1}\left(\pi\left(x^{*}\right)\right)$ for some $C_{0}$ universal. This implies

$$
z^{*}=t e_{n} \quad \text { with } \quad t \in\left[-\frac{R}{8}-\frac{C_{1}}{R},-\frac{R}{8}+\frac{C_{1}}{R}\right] .
$$

Moreover, $\pi\left(x^{*}\right) \in B_{C_{2}}$ since otherwise $\pi\left(x^{*}\right)$ is at a distance greater than $\frac{1}{R} \frac{C_{2}^{2}}{8}>$ $\frac{C_{1}}{R}$ in the interior of the ball $B_{R}\left(-R e_{n}\right)$, hence $\{u=0\}$ must intersect this ball and we reach a contradiction.

Now we apply Lemma 4.3 and Remark 4.4 at $\pi\left(x^{*}\right)$ and obtain the conclusion of the lemma.

It remains to show that $u<\phi_{R / 8,-R e_{n}}$. By hypothesis b) and Harnack inequality we see that $u$ is still sufficiently close to -1 in a whole ball $B_{R_{0}}\left(x_{0}\right)$ for some large universal $R_{0}$, and therefore $u<\phi_{R_{0} / 2, x_{0}}$ provided that $c$ is sufficiently small. Now we deform $\Phi_{R_{0} / 2, x_{0}}$ by a continuous family of functions $\Phi_{r, z}$ and first we move $z$ continuously from $x_{0}$ to $-R e_{n}$ and then we increase the radius $r$ from $R_{0}$ to $R / 8$. By Lemma 4.3 the graphs of these functions cannot touch the graph of $U$ by above and we obtain the desired inequality. With this the lemma is proved.

In the next lemma we prove a localized version of Lemma 4.5

Proposition 4.6. Assume that $U$ satisfies the equation in $\mathcal{B}_{R^{1-\sigma}}$ with $\sigma$ small, universal as in Lemma 3.2, and

a) $B_{R}\left(-R e_{n}\right) \cap B_{R^{\frac{1}{2}-\sigma}} \subset\{u<0\}$ is tangent to $\{u=0\}$ at 0 ,

b) all balls of radius $\frac{1}{4} R^{1-\sigma}$ which are tangent by below to $\partial B_{R}\left(-R e_{n}\right)$ in $B_{R^{\frac{1}{2}-\sigma}}$ are included in $\{u<0\}$,

c) there is $x_{0} \in B_{R^{1-\sigma} / 4}\left(-\frac{1}{2} R^{1-\sigma} e_{n}\right)$ such that $u\left(x_{0}\right) \leq-1+c$.

Then in $B_{1}$ we have that $\{u=0\}$ is smooth and has curvatures bounded by $\frac{C}{R}$.

Proof. As in Lemma 4.5. we slide the graph of $\Phi_{R / 8, z}$ in the $e_{n}$ direction till it touches the graph of $U$, except that now we restrict only to the region

$$
\mathcal{C}_{R}:=\left\{\left|x^{\prime}\right| \leq \frac{1}{2} R^{\frac{1}{2}-\sigma}, \quad\left|x_{n}\right| \leq \frac{1}{2} R^{1-\sigma}, \quad|y| \leq \frac{1}{2} R^{1-\sigma}\right\} .
$$

In order to repeat the argument above we need to show that the first contact point is an interior point and it occurs in $\mathcal{C}_{R / 2}$. For this it suffices to prove that

$$
U<\Phi_{R / 8, z_{0}} \quad \text { in } \quad \mathcal{C}_{R} \backslash \mathcal{C}_{R / 2}, \quad z_{0}:=\left(-\frac{R}{8}+\frac{C_{1}}{R}\right) e_{n}
$$


We estimate $U$ by using the functions $\Psi_{R, z}$ given in Definition 4.2. Notice that Lemma 4.3 holds if we replace $\Phi_{R}$ by $\Psi_{R}$.

Now we slide the graphs $\Psi_{r, z}$ with $r:=\frac{1}{4} R^{1-\sigma}$ and $\left|z^{\prime}\right| \leq R^{\frac{1}{2}-\sigma}, z_{n}=-2 r$ upward in the $e_{n}$ direction. We use hypotheses b), c) and as in the proof of Lemma 4.5 we find $\Psi_{r, z}>U$ as long as $B_{r}(z)$ is at distance greater than $\mathrm{Cr}^{-1}$ from $\partial B_{R}\left(-R e_{n}\right)$. We obtain that

$$
U(x)<\Psi_{r}\left(d_{1}(x)+C r^{-1}, y\right),
$$

where $d_{1}(x)$ is the signed distance to $\partial B_{R}\left(-R e_{n}\right)$. From Remark 3.3 we have

$$
\Psi_{r}(s, y) \leq G_{R / 8}\left(s+(R / 8)^{-3 \sigma}, y\right) .
$$

We obtain

$$
U(x, y)<G_{R / 8}\left(d_{1}(x)+2 R^{-3 \sigma}, y\right) .
$$

Let $d_{2}(x)$ represent the distance to $\partial B_{R / 8}\left(z_{0}\right)$. Then in the region $\mathcal{C}_{R} \backslash \mathcal{C}_{R / 2}$ we have either

a) $\left|x^{\prime}\right| \geq \frac{1}{2}(R / 2)^{\frac{1}{2}-\sigma}$ and then

$$
d_{2}(x)-d_{1}(x) \geq-\frac{C_{1}}{R}+\frac{1}{R}\left|x^{\prime}\right|^{2} \geq 2 R^{-3 \sigma},
$$

or

b) $\min \left\{\left|x_{n}\right|, y\right\} \geq R^{1-\sigma} / 8$ and then both $\left(d_{2}(x), y\right)$ and $\left(d_{1}(x)+2 R^{-\sigma}, y\right)$ are outside $B_{1-\delta}^{+} \subset \mathbb{R}^{2}$, thus $G_{R / 8}$ at these two points has the same value.

From (4.5) we find

$$
U(x, y)<G_{R / 8}\left(d_{2}(x), y\right) \quad \text { in } \quad \mathcal{C}_{R} \backslash \mathcal{C}_{R / 2},
$$

and (4.3) is proved.

Next we consider the case in which the 0 level set of $u$ is tangent by above at the origin to the graph of a quadratic polynomial.

Proposition 4.7. Let $U$ satisfies the equation in $\mathcal{B}_{R^{1-\sigma}}$ and the hypothesis c) of Proposition 4.6. Assume the surface

$$
\Gamma:=\left\{x_{n}=\sum_{1}^{n-1} \frac{a_{i}}{2} x_{i}^{2}+b^{\prime} \cdot x^{\prime}\right\} \cap B_{R^{\frac{1}{2}-\sigma}} \quad \text { with } \quad\left|b^{\prime}\right| \leq \varepsilon, \quad\left|a_{i}\right| \leq \varepsilon^{-2} R^{-1},
$$

is tangent to $\{u=0\}$ at 0 for some small $\varepsilon$ that satisfies $\varepsilon \geq R^{-\sigma / 2}$, and assume further that all balls of radius $\frac{1}{2} R^{1-\sigma}$ which are tangent to $\Gamma$ by below are included in $\{u<0\}$. Then

$$
\sum_{1}^{n-1} a_{i} \leq C R^{-1}
$$

Proposition 4.7 states that the blow-down of $\{u=0\}$ satisfies the minimal surface equation in some viscosity sense. Indeed, if we take $\varepsilon=R^{-\sigma / 2}$, then the set $R^{\sigma-1}\{u=0\}$ cannot be touched at 0 in a $R^{-1 / 2}$ neighborhood of the origin by a surface with curvatures bounded by $1 / 2$ and mean curvature greater than $C R^{-\sigma}$. 
Proof. We argue as in the proof of Proposition 4.6] except that now we replace $\partial B_{R}\left(-R e_{n}\right)$ by $\Gamma$ and $\partial B_{R / 8}\left(z_{0}\right)$ by

$$
\Gamma_{2}:=\left\{x_{n}=\sum_{1}^{n-1} \frac{a_{i}}{2} x_{i}^{2}+b^{\prime} \cdot x^{\prime}+\frac{C_{1}}{R}-\frac{1}{R}\left|x^{\prime}\right|^{2}\right\} .
$$

We claim that

$$
U(x, y)<G_{R / 8}\left(d_{2}(x), y\right) \quad \text { in } \quad \mathcal{C}_{R} \backslash \mathcal{C}_{R / 2},
$$

where $d_{2}$ represents the signed distance to the $\Gamma_{2}$ surface and $\mathcal{C}_{R}$ is defined in (4.2). Using the surfaces $\Psi_{r, z}$ as comparison functions we obtain as in (4.4), (4.5) above that

$$
U(x, y)<G_{R / 8}\left(d_{1}(x)+C^{\prime} r^{-1}, y\right) \text { in } \mathcal{C}_{R}
$$

with $d_{1}(x)$ representing the signed distance to $\Gamma$. Notice that (4.6) is valid in our setting. Now we argue as in (4.7) and obtain the desired claim (4.8).

Next we show that $G_{R / 8}\left(d_{2}(x), y\right)$ is a supersolution away from the set $\left\{\left|d_{2}\right| \leq\right.$ $1, y=0\}$ provided that

$$
\sum_{1}^{n-1} a_{i} \geq M R^{-1}
$$

for some $M$ large, universal to be made precise later. The boundary inequality on $\{y=0\}$ is clearly satisfied and on $\{y>0\}$ we have

$$
\triangle_{a} G_{R / 8}\left(d_{2}(x), y\right)=\triangle_{a} G_{R / 8}(s, y)+H(x) \partial_{s} G_{R / 8}(s, y), \quad s:=d_{2}(x),
$$

where $H(x)$ represents the mean curvature at $x$ of the parallel surface to $\Gamma_{2}$, and $\triangle_{a}$ on the right hand side is with respect to the variables $(s, y)$. If $|s|>R^{1-\delta}$ then $\partial_{s} G_{R / 8}=0$, and if $|s| \leq R^{1-\delta}$ we show below that $H<0$, and in both cases we obtain $\triangle_{a} G_{R / 8} \leq 0$.

Let $\kappa_{i}, i=1, . ., n-1$, be the principal curvatures of $\Gamma_{2}$ at the projection of $x$ onto $\Gamma_{2}$. Notice that at this point the slope of the tangent plane to $\Gamma_{2}$ is less than $4 \varepsilon$ hence we have

$$
\left|\kappa_{i}\right| \leq 2 \varepsilon^{-2} R^{-1} \leq 2 R^{\sigma-1}, \quad \sum \kappa_{i} \leq-\sum a_{i}+C \varepsilon^{2} \max \left|a_{i}\right| \leq-\frac{1}{2} M R^{-1} .
$$

When $\left|d_{2}\right| \leq R^{1-\delta}$, we obtain $d_{2} \kappa_{i}=o(1), d_{2} \kappa_{i}^{2}=o\left(R^{-1}\right)$ (since $\sigma<\delta / 3$ ), hence

$$
H(x)=\sum \frac{\kappa_{i}}{1-d_{2} \kappa_{i}}=\sum\left(\kappa_{i}+\frac{d_{2} \kappa_{i}^{2}}{1-d_{2} \kappa_{i}}\right) \leq-\frac{1}{4} M R^{-1} .
$$

Now we translate the graph of $G_{R / 8}\left(d_{2}, y\right)$ along the $e_{n}$ direction till it touches the graph of $U$ by above. Precisely, we consider the graphs of $G_{R}\left(d_{2}\left(x-t e_{n}\right), y\right)$ with $t \leq 0$ and start with $t$ negative so that the function is identically 1 in $\mathcal{C}_{R}$. Then we increase $t$ continuously till this graph becomes tangent by above to the graph of $U$ in $\mathcal{C}_{R}$. Since $u(0)=0$, a contact point must occur for some $t \leq 0$ and, by (4.8), this point is interior to $\mathcal{C}_{R / 2}$ and lies on $y=0$. Let $\left(x^{*}, 0, u\left(x^{*}\right)\right)$ be the first contact point where a translate $G_{R / 8}\left(d_{2}\left(x-t^{*} e_{n}\right), y\right)$ touches $U$ by above. We show that we reach a contradiction if $M$ is chosen sufficiently large.

Define $V$ as

$$
V(x, y):=G\left(d_{2}\left(x-t^{*} e_{n}\right)+C / R, y\right) \geq G_{R / 8}\left(d_{2}\left(x-t^{*} e_{n}\right), y\right) \geq U(x, y) .
$$

Notice that

$$
\partial_{y}^{1-a} V=W^{\prime}(V), \quad(V-U)\left(x^{*}, 0\right) \leq C / R .
$$


In $\mathcal{B}_{1}\left(x^{*}\right)$ we use the computation (4.9) above for $V$ together with (4.10) and obtain

$$
\triangle_{a} V \leq-c M R^{-1}, \quad \text { in } \quad \mathcal{B}_{1}\left(x^{*}\right) .
$$

The function $Q:=(V-U) /\left(c M R^{-1}\right) \geq 0$ satisfies in $\mathcal{B}_{1}\left(x^{*}\right)$

$$
\triangle_{a} Q \leq-1, \quad\left|\partial_{y}^{1-a} Q\right| \leq C Q, \quad Q\left(x^{*}, 0\right) \leq C^{\prime} M^{-1} .
$$

By the maximum principle

$$
Q(x, y) \geq \mu^{2}+\mu y^{1-a}-\frac{1}{2(n+1)}\left(\left|x-x^{*}\right|^{2}+y^{2}\right),
$$

for some $\mu$ small universal, and we reach a contradiction at $\left(x^{*}, 0\right)$ if $M$ is sufficiently large.

\section{HARNACK INEQUALITY}

In this section we use Proposition 4.6 and prove a Harnack inequality property for flat level sets, see Theorem 5.1 below. The key step in the proof is to control the $x_{n}$ coordinate of the level set $\{u=0\}$ in a set of large measure in the $x^{\prime}$-variables.

Notation: We denote by $\mathcal{C}(l, \theta)$ the cylinder

$$
\mathcal{C}(l, \theta):=\left\{\left|x^{\prime}\right| \leq l, \quad\left|x_{n}\right| \leq \theta\right\} .
$$

Theorem 5.1 (Harnack inequality for minimizers). Let $U$ be a minimizer of $J$ in $\mathcal{B}_{q}$ and assume that

$$
0 \in\{u=0\} \cap \mathcal{C}(l, l) \subset \mathcal{C}(l, \theta),
$$

and that all balls of radius $q:=\left(l^{2} \theta^{-1}\right)^{1-\frac{\sigma}{2}}$ which are tangent to $\mathcal{C}(l, \theta)$ by below and above are included in $\{u<0\}$ respectively $\{u>0\}$.

Given $\theta_{0}>0$ there exist $\omega>0$ small depending on $n, W$, and $\varepsilon_{0}\left(\theta_{0}\right)>0$ depending on $n, W$ and $\theta_{0}$, such that if

$$
\theta l^{-1} \leq \varepsilon_{0}\left(\theta_{0}\right), \quad \theta_{0} \leq \theta,
$$

then

$$
\{u=0\} \cap \mathcal{C}(\bar{l}, \bar{l}) \subset \mathcal{C}(\bar{l}, \bar{\theta}), \quad \bar{l}:=l / 4, \quad \bar{\theta}:=(1-\omega) \theta,
$$

and all balls of radius $\bar{q}:=\left(\bar{l}^{2} \bar{\theta}^{-1}\right)^{1-\frac{\sigma}{2}}$ which are tangent to $\mathcal{C}(\bar{l}, \bar{\theta})$ by below or above do not intersect $\{u=0\}$.

The fact that $u$ is a minimizer of $J$ is only used in a final step of the proof. This hypothesis can be replaced by $x_{n}$ monotonicity for $u$, or more generally by the monotonicity of $u$ in a given direction which is not perpendicular to $e_{n}$.

Definition 5.2. For a small $a>0$, we denote by $\mathcal{D}_{a}$ the set of points on

$$
\{u=0\} \cap \mathcal{C}\left(\frac{3}{4} l, \theta\right)
$$

which have a paraboloid of opening $-a$ and vertex $y=\left(y^{\prime}, y_{n}\right)$

$$
P_{a, y}:=\left\{x_{n}=-\frac{a}{2}\left|x^{\prime}-y^{\prime}\right|^{2}+y_{n}\right\}
$$

tangent by below in $\mathcal{C}(l, \theta)$, and with $P_{a, y}$ below the lateral boundary of $\mathcal{C}(l, \theta)$. In other words we allow only those polynomials $P_{a, y}$ which exit $\mathcal{C}(l, \theta)$ through the "bottom".

We denote by $D_{a} \subset \mathbb{R}^{n-1}$ the projection of $\mathcal{D}_{a}$ into $\mathbb{R}^{n-1}$ along the $e_{n}$ direction. 
By Proposition 4.6 we see that as long as

$$
l^{-1} \geq a \geq l^{-2-\eta}, \quad \text { and } \quad l \geq C\left(\theta_{0}\right),
$$

for some $\eta$ small universal (depending on $\sigma$ ), then $\{u=0\}$ has the following property $(P)$ :

$(P)$ In a neighborhood of any point of $\mathcal{D}_{a}$ the set $\{u=0\}$ is a graph in the $e_{n}$ direction of a $C^{2}$ function with second derivatives bounded by $\Lambda a$ with $\Lambda$ a universal constant.

Indeed, since $a \leq l^{-1}$, at a point $z \in \mathcal{D}_{a}$ the corresponding paraboloid at $z$ has a tangent ball of radius

$$
R:=c a^{-1} \leq l^{2+\eta}
$$

by below. Since $\left|z^{\prime}\right| \leq 3 / 4 l$ we see that $\{u=0\} \cap B_{l / 4}(z)$ has a tangent ball $B_{R}\left(x_{0}\right)$ by below at $z$ and hypothesis a) of Proposition 4.6 holds since

$$
l / 4 \geq R^{\frac{1}{2}-\sigma} .
$$

The assumption that all balls of radius $q \geq c\left(\theta_{0}\right) l^{2-\sigma} \geq R^{1-\sigma}$ tangent by below to $C(l, \theta)$ are included in $\{u<0\}$ gives that all balls tangent to $\partial B_{R}\left(x_{0}\right) \cap B_{l / 4}(z)$ by below are also included in $\{u<0\}$ hence hypothesis b) of Proposition 4.6 holds.

Since $u$ is a minimizer, in any sufficiently large ball in $\{u<0\}$ we have points that satisfy $u<-1+c$ and hypothesis c) holds as well. In conclusion Proposition 4.6 applies and property $(P)$ holds.

Since $\{u=0\}$ satisfies property $(P)$ then it satisfies a general version of Weak Harnack inequality which we proved in $\mathrm{S2}$. In particular we are in the setting of Propositions 6.2 and 6.4 (see also Remark 6.7) in [S2].

This means that for any $\mu>0$ small, there exists $M(\mu)$ depending on $\mu$ and universal constants such that if

$$
\{u=0\} \cap\left(B_{l / 2}^{\prime} \times[-\theta,(\omega-1) \theta]\right) \neq \emptyset, \quad \omega:=(32 M)^{-1},
$$

then, by Proposition 6.2 in [S2], we obtain

$$
\mathcal{H}^{n-1}\left(D_{a} \cap B_{l / 2}^{\prime}\right) \geq(1-\mu) \mathcal{H}^{n-1}\left(B_{l / 2}^{\prime}\right), \quad \text { with } \quad a:=M \omega \theta l^{-2},
$$

and

$$
\mathcal{D}_{a} \cap\left\{\left|x^{\prime}\right| \leq l / 2\right\} \subset\left\{x_{n} \leq(8 M \omega-1) \theta\right\}=\left\{x_{n} \leq-3 \theta / 4\right\} .
$$

We can apply Proposition 6.2 in $S 2$ since the interval $I$ of allowed openings of the paraboloids satisfies (see (5.1))

$$
I=\left[\omega \theta l^{-2}, M \omega \theta l^{-2}\right] \subset\left[l^{-2-\eta}, l^{-1}\right],
$$

provided that $l \geq C\left(\mu, \theta_{0}\right)$ and $\varepsilon_{0} \leq c$.

Next we let $\mathcal{D}_{a}^{*}$ to denote the set of points on

$$
\mathcal{D}_{a}^{*}:=\{u=0\} \cap\left(\left\{\left|x^{\prime}\right| \leq l / 2\right\} \times\left[-\frac{1}{2} \theta, \theta\right]\right)
$$

which admit a tangent paraboloid of opening $a$ by above which exists $\mathcal{C}(l, \theta)$ through the "top". Also we denote by $D_{a}^{*} \subset \mathbb{R}^{n-1}$ the projection of $\mathcal{D}_{a}^{*}$ along $e_{n}$. Then according to Proposition 6.4 in [S2], (applied "up-side down") we have

$$
\mathcal{H}^{n-1}\left(D_{\tilde{a}}^{*} \cap B_{l / 2}^{\prime}\right) \geq \mu_{0} \mathcal{H}^{n-1}\left(B_{l / 2}^{\prime}\right), \quad \text { with } \quad \tilde{a}=8 \theta l^{-2},
$$


for some $\mu_{0}$ universal.

We choose $\mu$ in (5.2)-(5.4) universal as

$$
\mu:=\mu_{0} / 2 .
$$

According to (5.3), (5.6) this gives

$$
\mathcal{H}^{n-1}\left(D_{a} \cap D_{\tilde{a}}^{*}\right) \geq \frac{\mu_{0}}{2} \mathcal{H}^{n-1}\left(B_{l / 2}^{\prime}\right) .
$$

Notice that by (5.4), (5.5) the sets $\mathcal{D}_{a}$ and $\mathcal{D}_{\tilde{a}}^{*}$ are disjoint.

At this point we would reach a contradiction (to (5.2) ) if $\{u=0\}$ were assumed to be a graph in the $e_{n}$ direction. Instead we use (5.7) and show that $U$ cannot be a minimizer.

Proof of Theorem 5.1.

It suffices to show that

$$
\{u=0\} \cap \mathcal{C}(l / 2, l / 2) \subset C(l / 2,(1-\omega) \theta) .
$$

Then the existence of the balls of size $q \ll l^{2} \theta^{-1}$ (included in $\{u<0\}$ and $\{u>$ $0\}$ respectively) tangent to $C(l / 4,(1-\omega) \theta)$ follows easily as we restrict from the cylinder of size $l / 2$ to the one of size $l / 4$, and the conclusion is satisfied since $\tilde{q} \leq q$.

Assume by contradiction that (5.2) holds, and therefore (5.3), (5.7) hold as well. For each $x \in \mathcal{D}_{a}$ the set $\{u=0\}$ has a tangent ball of radius $c a^{-1} \geq c l$ by below. Moreover, the normal to this balls at the contact points and the $e_{n}$ direction make a small angle which is bounded by $c \theta l^{-1} \leq c \varepsilon_{0}$. According to Lemma 4.5 part 2) and Remark 4.4, we conclude that for any fixed constant $K$ we have

$$
\max _{(t, y) \in \mathcal{B}_{K}^{+}}\left|U\left(x^{\prime}, x_{n}+t, y\right)-G(t, y)\right| \leq \rho,
$$

with $\rho=\rho\left(K, \varepsilon_{0}\right) \rightarrow 0$ as $\varepsilon_{0} \rightarrow 0$.

We denote the $2 \mathrm{D}$ half disk of radius $r$ in the $\left(x_{n}, y\right)$-variables centered at $z \in \mathbb{R}^{n}$ as

$$
\mathcal{B}_{r, z}^{+}:=\left\{\left(z^{\prime}, z_{n}+t, y\right)|\quad|(t, y) \mid \leq r, \quad y \geq 0\right\} .
$$

From above we find for all $x \in \mathcal{D}_{a}$, or similarly if $x \in \mathcal{D}_{a}^{*}$, we have

$$
J\left(U, \mathcal{B}_{K, x}^{+}\right) \geq \mathcal{J}\left(G, \mathcal{B}_{K}^{+}\right)-\bar{\rho},
$$

with $\bar{\rho}=\bar{\rho}\left(K, \varepsilon_{0}\right) \rightarrow 0$ as $\varepsilon_{0} \rightarrow 0$.

If $x^{\prime} \in D_{a} \cap D_{\tilde{a}}^{*}$ then by (5.4), (5.5) the two points $x^{1}=\left(x^{\prime}, x_{n}^{1}\right) \in \mathcal{D}_{a}$ and $x^{2}=\left(x^{\prime}, x_{n}^{2}\right) \in \mathcal{D}_{\tilde{a}}^{*}$ satisfy $x_{n}^{2}-x_{n}^{1} \geq \theta / 4 \geq \theta_{0} / 4$. By (5.8) this means that the two disks $\mathcal{B}_{K, x^{i}}$ are disjoint provided that $\rho$ is small, thus

$$
\mathcal{J}\left(U, \mathcal{B}_{l / 2,\left(x^{\prime}, 0\right)}^{+}\right) \geq 2\left(\mathcal{J}\left(G, \mathcal{B}_{K}^{+}\right)-\bar{\rho}\right) \quad \text { if } \quad x^{\prime} \in D_{a} \cap D_{\tilde{a}}^{*} .
$$

We integrate in $x^{\prime}$ and use also (5.3), (5.7), (5.9) to obtain

$$
\mathcal{J}\left(U, A_{l / 2}\right) \geq\left(1+\mu_{0} / 2\right)\left(\mathcal{J}\left(G, \mathcal{B}_{K}^{+}\right)-\bar{\rho}\right) \mathcal{H}^{n-1}\left(B_{l / 2}^{\prime}\right),
$$

with

$$
A_{l / 2}:=\mathcal{C}\left(\frac{l}{2}, \frac{l}{2}\right) \times\left[0, \frac{l}{2}\right] .
$$

We choose first $K$ large and then $\varepsilon_{0}$ small such that $\bar{\rho}$ is sufficiently small such that

$$
\mathcal{J}\left(U, A_{l / 2}\right) \geq\left(1+\mu_{0} / 4\right) \mathcal{J}\left(G, \mathbb{R}_{+}^{2}\right) \mathcal{H}^{n-1}\left(B_{l / 2}^{\prime}\right) .
$$


This contradicts Lemma 5.3 below provided that $\varepsilon_{0}$ is taken sufficiently small.

Next lemma is a $\Gamma$-convergence result and it is a consequence of the minimality of $U$ in $A_{l / 2}$.

Lemma 5.3.

$$
\mathcal{J}\left(U, A_{l / 2}\right) \leq \mathcal{J}\left(G, \mathbb{R}_{+}^{2}\right) \mathcal{H}^{n-1}\left(B_{l / 2}^{\prime}\right)+\gamma\left(\varepsilon_{0}\right) l^{n-1} .
$$

with $\gamma\left(\varepsilon_{0}\right) \rightarrow 0$ as $\varepsilon_{0} \rightarrow 0$.

Proof. We interpolate between $U$ and $V(x, y):=G\left(x_{n}, y\right)$ as

$$
H=(1-\varphi) U+\varphi V, .
$$

Here $\varphi$ is a cutoff Lipschitz function such that $\varphi=0$ outside $A_{l / 2}, \varphi=1$ in $\mathcal{R}$ and $|\nabla \varphi| \leq 8 /(1+y)$ in $A_{l / 2} \backslash \mathcal{R}$, where $\mathcal{R}$ is the cone

$$
\mathcal{R}:=\{(x, y)|\quad| x \mid \leq l / 2-1-2 y\} .
$$

By minimality of $U$ we have

$$
\mathcal{J}\left(U, A_{l / 2}\right) \leq \mathcal{J}\left(H, A_{l / 2}\right)=\mathcal{J}(V, \mathcal{R})+\mathcal{J}\left(H, A_{l / 2} \backslash \mathcal{R}\right)
$$

Since

$$
\mathcal{J}(V, \mathcal{R}) \leq \mathcal{J}\left(V, A_{l / 2}\right) \leq \mathcal{J}\left(G, \mathbb{R}_{+}^{2}\right) \mathcal{H}^{n-1}\left(B_{l / 2}^{\prime}\right)
$$

we need to show that

$$
\mathcal{J}\left(H, A_{l / 2} \backslash \mathcal{R}\right) \leq \gamma l^{n-1}
$$

with $\gamma$ arbitrarily small. We have

$$
\begin{gathered}
\mathcal{J}\left(H, A_{l / 2} \backslash \mathcal{R}\right) \leq 4 \int_{A_{l / 2} \backslash \mathcal{R}}\left(|\nabla \varphi|^{2}(V-U)^{2}+|\nabla(V-U)|^{2}\right) y^{a} d x d y \\
\quad+\int_{B_{l / 2} \backslash B_{l / 2-1}}(v-u)^{2} d x .
\end{gathered}
$$

We use that $|U|,|V| \leq 1,|\nabla U|,|\nabla V| \leq C /(1+y)$ and we see that in (5.12) the first integral in the region where $y \geq C \gamma^{1 / a}$ is bounded by

$$
\int_{C \gamma^{1 / a}}^{l / 2} C_{1}(1+y)^{-2}(1+y) y^{a} d y \leq \gamma / 4 \text {. }
$$

Next we notice that $u$ and $v$ are sufficiently close to each other in $\mathcal{C}(l / 2, l / 2)$ away from a thin strip around $x_{n}=0$. Indeed, we can use barrier functions as in Proposition 4.6 (see (4.4) ) and bound $u$ by above an below in terms of the function $\psi_{l / 2}$ and distance to the hyperplanes $x_{n}= \pm \theta$. This implies that

$$
|v-u| \leq \gamma \quad \text { in } \mathcal{C}(l / 2, l / 2) \text { if }\left|x_{n}\right| \geq C(\gamma)+\theta
$$

with $C(\gamma)$ large, depending on the universal constants and $\gamma$. For the extensions $U$ and $V$ this gives

$$
|V-U|,|\nabla(V-U)| \leq C_{2} \gamma \quad \text { in } A_{l / 2} \text { if }\left|x_{n}\right| \geq C^{\prime}(\gamma)+\theta \text { and } y \leq C \gamma^{1 / a}
$$

with $C_{2}$ universal. Now (5.11) easily follows from (5.12). 


\section{IMPROVEMENT OF FLATNESS}

We state the improvement of flatness property of minimizers.

Theorem 6.1 (Improvement of flatness). Let $U$ be a minimizer of $J$ in $\mathcal{B}_{q}$ and assume that

$$
0 \in\{u=0\} \cap \mathcal{C}(l, l) \subset \mathcal{C}(l, \theta),
$$

and that all balls of radius $q:=\left(l^{2} \theta^{-1}\right)^{1-\frac{\sigma}{2}}$ which are tangent to $\mathcal{C}(l, \theta)$ by below and above are included in $\{u<0\}$ respectively $\{u>0\}$.

Given $\theta_{0}>0$ there exist $\eta>0$ small depending on $n$, and $\varepsilon_{1}\left(\theta_{0}\right)>0$ depending on $n, W$ and $\theta_{0}$, such that if

$$
\theta l^{-1} \leq \varepsilon_{1}\left(\theta_{0}\right), \quad \theta_{0} \leq \theta,
$$

then

$$
\{u=0\} \cap \mathcal{C}_{\xi}(\bar{l}, \bar{l}) \subset C_{\xi}(\bar{l}, \bar{\theta}), \quad \bar{l}:=\eta l, \quad \bar{\theta}:=\eta^{3 / 2} \theta,
$$

and all balls of radius $\bar{q}:=\left(\bar{l}^{2} \bar{\theta}^{-1}\right)^{1-\frac{\sigma}{2}}$ which are tangent to $\mathcal{C}_{\xi}(\bar{l}, \bar{\theta})$ by below and above are included in $\{u<0\}$ respectively $\{u>0\}$.

Here $\xi \in \mathbb{R}^{n}$ is a unit vector and $C_{\xi}(\bar{l}, \bar{\theta})$ represents the cylinder with axis $\xi$, base $\bar{l}$ and height $\bar{\theta}$.

As a consequence of this flatness theorem we obtain our main theorem.

Theorem 6.2. Let $U$ be a global minimizer of J. Suppose that the 0 level set $\{u=0\}$ is asymptotically flat at $\infty$, i.e there exist sequences of positive numbers $\theta_{k}, l_{k}$ and unit vectors $\xi_{k}$ with $l_{k} \rightarrow \infty, \theta_{k} l_{k}^{-1} \rightarrow 0$ such that

$$
\{u=0\} \cap B_{l_{k}} \subset\left\{\left|x \cdot \xi_{k}\right|<\theta_{k}\right\} .
$$

Then the 0 level set is a hyperplane and $u$ is one-dimensional.

By saying that $u$ is one-dimensional we understand that $u$ depends only on one direction $\xi$, i.e $u=g(x \cdot \xi)$.

Proof. Without loss of generality assume $u(0)=0$. Fix $\theta_{0}>0$, and $\varepsilon \leq \varepsilon_{1}\left(\theta_{0}\right)$. We choose $k$ sufficiently large such that, after increasing $\theta_{k}$ if necessary we have $\theta_{k} l_{k}^{-1}=\varepsilon$. We can apply Theorem 6.1 since $q=\left(l_{k} \varepsilon^{-1}\right)^{1-\frac{\sigma}{2}} \ll l_{k}$, and we obtain that $\{u=0\}$ is trapped in a flatter cylinder. We apply Theorem 6.1 repeatedly till the height of the cylinder becomes less than $\theta_{0}$. We conclude that $\{u=0\}$ is trapped in a cylinder withe flatness less than $\varepsilon$ and height $\theta_{0}$. We let first $\varepsilon \rightarrow 0$ and then $\theta_{0} \rightarrow 0$ and obtain the desired conclusion.

Proof of Theorem 6.1

The proof is by compactness and it follows from Theorem 5.1 and Proposition 4.7 Assume by contradiction that there exist $U_{k}, \theta_{k}, l_{k}, \xi_{k}$ such that $u_{k}$ is a minimizer of $J, u_{k}(0)=0$, and the level set $\left\{u_{k}=0\right\}$ stays in the flat cylinder $\mathcal{C}\left(l_{k}, \theta_{k}\right)$ with $\theta_{k} \geq \theta_{0}, \theta_{k} l_{k}^{-1} \rightarrow 0$ as $k \rightarrow \infty$ for which the conclusion of Theorem 6.1 doesn't hold.

Let $A_{k}$ be the rescaling of the 0 level sets given by

$$
\begin{gathered}
\left(x^{\prime}, x_{n}\right) \in\left\{u_{k}=0\right\} \mapsto\left(z^{\prime}, z_{n}\right) \in A_{k} \\
z^{\prime}=x^{\prime} l_{k}^{-1}, \quad z_{n}=x_{n} \theta_{k}^{-1} .
\end{gathered}
$$


Claim 1: $A_{k}$ has a subsequence that converges uniformly on $\left|z^{\prime}\right| \leq 1 / 2$ to a set $A_{\infty}=\left\{\left(z^{\prime}, w\left(z^{\prime}\right)\right), \quad\left|z^{\prime}\right| \leq 1 / 2\right\}$ where $w$ is a Holder continuous function. In other words, given $\varepsilon$, all but a finite number of the $A_{k}$ 's from the subsequence are in an $\varepsilon$ neighborhood of $A_{\infty}$.

Proof: Fix $z_{0}^{\prime},\left|z_{0}^{\prime}\right| \leq 1 / 2$ and suppose $\left(z_{0}^{\prime}, z_{k}\right) \in A_{k}$. We apply Theorem 5.1 for the function $u_{k}$ in the cylinder

$$
\left\{\left|x^{\prime}-l_{k} z_{0}^{\prime}\right|<l_{k} / 2\right\} \times\left\{\left|x_{n}-\theta_{k} z_{k}\right|<2 \theta_{k}\right\}
$$

in which the set $\left\{u_{k}=0\right\}$ is trapped. Thus, there exist an increasing function $\varepsilon_{0}(\theta)>0, \varepsilon_{0}(\theta) \rightarrow 0$ as $\theta \rightarrow 0$, such that $\left\{u_{k}=0\right\}$ is trapped in the cylinder

$$
\left\{\left|x^{\prime}-l_{k} z_{0}^{\prime}\right|<l_{k} / 8\right\} \times\left\{\left|x_{n}-\theta_{k} z_{k}\right|<2(1-\omega) \theta_{k}\right\}
$$

provided that $4 \theta_{k} l_{k}^{-1} \leq \varepsilon_{0}\left(2 \theta_{k}\right)$. Rescaling back we find that

$$
A_{k} \cap\left\{\left|z^{\prime}-z_{0}^{\prime}\right| \leq 1 / 8\right\} \subset\left\{\left|z_{n}-z_{k}\right| \leq 2(1-\omega)\right\} .
$$

We apply the Harnack inequality repeatedly and we find that

$$
A_{k} \cap\left\{\left|z^{\prime}-z_{0}^{\prime}\right| \leq 2^{-2 m-1}\right\} \subset\left\{\left|z_{n}-z_{k}\right| \leq 2(1-\omega)^{m}\right\}
$$

provided that

$$
\theta_{k} l_{k}^{-1} \leq 4^{-m-1} \varepsilon_{0}\left(2(1-\omega)^{m} \theta_{k}\right) .
$$

Since these inequalities are satisfied for all $k$ large we conclude that (6.1) holds for all but a finite number of $k$ 's. Now the claim follows from Arzela-Ascoli Theorem.

Claim 2: The function $w$ is harmonic (in the viscosity sense).

Proof: The proof is by contradiction. Fix a quadratic polynomial

$$
z_{n}=P\left(z^{\prime}\right)=\frac{1}{2} z^{\prime T} M z^{\prime}+\xi \cdot z^{\prime}, \quad\|M\|<\delta^{-1}, \quad|\xi|<\delta^{-1}
$$

such that $\operatorname{tr} M>\delta, P\left(z^{\prime}\right)+\delta\left|z^{\prime}\right|^{2}$ touches the graph of $w$, say, at 0 for simplicity, and stays below $w$ in $\left|z^{\prime}\right|<8 \delta$, for some small $\delta$. Notice that at all points in the cylinder $\left|z^{\prime}\right|<2 \delta$, the quadratic polynomial above admits a tangent paraboloid by below of opening $-\delta^{-2}$ which is below $z_{n}=-2$ when $\left|z^{\prime}\right| \geq 6 \delta$.

Thus, for all $k$ large we find points $\left(z_{k}{ }^{\prime}, z_{k_{n}}\right)$ close to 0 such that $P\left(z^{\prime}\right)+$ const touches $A_{k}$ by below at $\left(z_{k}{ }^{\prime}, z_{k n}\right)$ and stays below it in $\left|z^{\prime}-z_{k}{ }^{\prime}\right|<\delta$.

This implies that, after eventually a translation, there exists a surface

$$
\Gamma:=\left\{x_{n}=\frac{\theta_{k}}{l_{k}^{2}} \frac{1}{2} x^{\prime T} M x^{\prime}+\frac{\theta_{k}}{l_{k}} \xi_{k} \cdot x^{\prime}\right\}, \quad\left|\xi_{k}\right|<2 \delta^{-1}
$$

that touches $\left\{u_{k}=0\right\}$ at the origin and stays below it in $\mathcal{C}\left(\delta l_{k}, 2 \theta_{k}\right)$. Moreover in the cylinder $\mathcal{C}\left(l_{k} / 2,2 \theta_{k}\right)$ the surface $\Gamma$ admits at all points with $\left|x^{\prime}\right| \leq \delta l$ a tangent ball by below of radius $\delta^{2} l_{k}^{2} \theta_{k}^{-1} \gg q$. In view of our hypothesis we conclude that $\Gamma \cap B_{\delta l_{k}}$ admits at all its points a tangent ball of radius $q$ by below which is included in $\{u<0\}$.

We contradict Proposition 4.7 by choosing $R$ as

$$
R^{-1}:=C^{-1} \delta \theta_{k} l_{k}^{-2},
$$


with $C$ the constant from Proposition 4.7 and with $\varepsilon=\delta^{2}$. Then for all large $k$ we have

$$
\theta_{k} l_{k}^{-1}\left|\xi_{k}\right| \leq \varepsilon, \quad \theta_{k} l_{k}^{-2}\|M\| \leq \varepsilon^{-2} R^{-1}, \quad \delta l_{k} \geq R^{\frac{1}{2}-\sigma}, \quad q \geq R^{1-\sigma},
$$

and Proposition 4.7 applies. We obtain $\operatorname{tr} M \leq \delta$ and we reached a contradiction.

Since $w$ is harmonic, there exist $0<\eta$ small depending only on $n$ such that

$$
\left|w-\xi \cdot z^{\prime}\right|<\frac{1}{2} \eta^{3 / 2} \quad \text { for }\left|z^{\prime}\right|<2 \eta,
$$

and the parabolas of opening $-C$ tangent by below (and above) to

$$
z_{n}=\xi \cdot z^{\prime} \pm \frac{1}{2} \eta^{3 / 2}
$$

in the cylinder $\left|z^{\prime}\right|<2 \eta$ lie below (or above) to the graph of $w$.

Rescaling back and using the fact that $A_{k}$ converge uniformly to the graph of $w$ and that $\bar{q}<q$ we easily conclude that $u_{k}$ satisfies the conclusion of the Theorem 6.1 for $k$ large enough, and we reached a contradiction.

\section{REFERENCES}

[CC1] Cabre X., Cinti E., Energy estimates and 1-D symmetry for nonlinear equations involving the half- Laplacian. Discrete Contin. Dyn. Syst.28 , 1179-1206 (2010).

[CC2] Cabre X., Cinti E., Sharp energy estimates for nonlinear fractional diffusion equations. Calc. Var. Partial Differential Equations 49 (2014), no. 1-2, 233-269.

[CS1] Cabre X., Sire Y., Nonlinear equations for fractional Laplacians I: regularity, maximum principles, and Hamiltonian estimates. Ann. Inst. H. Poincare Anal. Non Linaire 31 (2014), no. 1, 23-53.

[CS2] Cabre X., Sire Y., Nonlinear equations for fractional Laplacians II: existence, uniqueness, and qualitative properties of solutions. Trans. Am. Math. Soc. 367 (2015), no. 2, 911-941.

[CSo] Cabre X., Sola-Morales J., Layer solutions in a half-space for boundary reactions. Commun. Pure Appl. Math. 58, 1678-1732 (2005)

[CS] Caffarelli L., Silvestre L., An extension problem related to the fractional Laplacian. Comm. Part. Diff. Eq. 32, 1245-1260 (2007)

[D] De Giorgi E., Convergence problems for functional and operators. Proc. Int. Meeting on Recent Methods in Nonlinear Analysis (Rome, 1978), 131-188.

[DVS] Dipierro S., Serra J., Valdinoci E., Improvement of flatness for nonlocal phase transitions. Preprint

[PSV] Palatucci G., Savin O., Valdinoci E., Local and global minimizers for a variational energy involving a fractional norm. Ann. Mat. Pura Appl. (4) 192 (2013), no. 4, 673-718.

[S1] Savin O., Regularity of flat level sets in phase transitions. Ann. of Math. (2) 169 (2009), no.1, 41-78.

[S2] Savin O., Some remarks on the classification of global solutions with asymptotically flat level sets arxiv 1610.03448

[SV1] Savin O., Valdinoci E., Density estimates for a variational model driven by the Gagliardo norm, J. Math. Pures Appl. (9) 101 (2014), no. 1, 1-26.

[SV2] Savin, O., Valdinoci E., -convergence for nonlocal phase transitions. Ann. Inst. H. Poincare Anal. Non Linaire 29 (2012), no. 4, 479-500.

[SiV] Sire, Y., Valdinoci, E.: Fractional Laplacian phase transitions and boundary reactions: a geometric inequality and a symmetry result. J. Funct. Anal. 256 (2009), no. 6, 1842-1864.

Department of Mathematics, Columbia University, New York, NY 10027

E-mail address: savin@math.columbia.edu 